\title{
Task-irrelevant blindsight and the impact of invisible stimuli
}

\author{
Petra Stoerig* \\ Institute of Experimental Psychology II, Heinrich-Heine-University Dusseldorf, Dusseldorf, Germany
}

\section{Edited by:}

Bernard J. Baars, The Neurosciences Institute, USA

\section{Reviewed by:}

Jochen Braun, University of

Magdeburg, Germany

Daniel Bor, University of Sussex, UK

*Correspondence:

Petra Stoerig, Institute of Experimental Psychology II, Heinrich-Heine-

University, Building 23.03,

Universitaetsstrasse 1, 40225

Dusseldorf, Germany.

e-mail: petra.stoerig@uni-duesseldorf.de
Despite their subjective invisibility, stimuli presented within regions of absolute cortical blindness can both guide forced-choice behavior when they are task-relevant and modulate responses to visible targets when they are task-irrelevant. We here tested three hemianopic patients to learn whether their performance in an attention-demanding rapid serial visual presentation task would be affected by task-irrelevant stimuli. Per trial, nine black letters and one white target letter appeared briefly at fixation; the white letter was to be named at the end of each trial. On $50 \%$ of trials, a task-irrelevant disk ( -0.6 log contrast) was presented to the blind field; in separate blocks, the same or a very low negative contrast distractor was presented to the sighted field. Mean error rates were high and independent of distractor condition, although the high-contrast sighted-field disk impaired performance significantly in one participant. However, when trials with and without distractors were considered separately, performance was most impaired by the high-contrast disk in the blind field, whereas the same disk in the sighted field had no effect. As this disk was least visible in the blind and most visible in the sighted field, attentional suppression was inversely related to visibility. We suggest that visual awareness, or the processes that generate it and are compromised in the blind hemisphere, enhances or enables effective attentional suppression.

Keywords: blindsight, rapid serial visual presentation, attentional suppression, target detection, function of conscious sight, primary visual cortex

\section{INTRODUCTION}

Lesions that destroy or denervate the primary visual cortex (V1, striate cortex, Brodmann Area 17) cause homonymous fields of cortical blindness in the contralesional hemifield (Inouye, 1909; Holmes, 1918; Teuber et al., 1960). If the blindness is absolute, stimuli presented within the blind field are subjectively invisible. Nevertheless, they can give rise to a variety of non-reflexive visual functions that include detection, localization, and discrimination of blind-field stimuli that differ in flux, orientation, motion, wavelength, facial expression (De Gelder et al., 1999), and, possibly, motion direction (Pizzamiglio et al., 1984; Perenin, 1991; Barton and Sharpe, 1997; Benson et al., 1998; Azzopardi and Cowey, 2001; Morland et al., 2004) and shape (Weiskrantz et al., 1974; Weiskrantz, 1987; Perenin and Rosetti, 1996; Trevethan et al., 2007; for reviews see Weiskrantz, 1986, 1990; Stoerig and Cowey, 1997; Cowey, 2010). These functions rely on the retinofugal pathways that, despite the effects of the lesion and the degeneration it provokes, continue to transmit visual information to the retinorecipient nuclei which, in turn, project it directly or indirectly to the extrastriate cortical areas (Pasik and Pasik, 1982; Cowey and Stoerig, 1991; Payne et al., 1996; Ptito et al., 1999; Goebel et al., 2001). They have become widely known as blindsight (Sanders et al., 1974; Weiskrantz et al., 1974), a term that captures the counterintuitive contrast between the experiential blindness and the often highly significant responses the stimuli evoke even under strictly controlled experimental conditions.

As the patients do not see the stimuli and thus cannot be asked to describe their location, color, or shape, blindsight has routinely been investigated with forced-choice methods that require the patients to guess whether, where, or which target was briefly presented to the blind field (Pöppel et al., 1973; Richards, 1973). Rather than requiring the participant to respond, usually by guessing, to the unseen targets, the alternative and less frequently used indirect approach engages the patient in responding to stimuli presented to the sighted field; processing of stimuli presented, unbeknownst to the patient, to the blind field, is then inferred from their effects on performance. Examples of such effects include faster response times to seen targets (Marzi et al., 1986; Corbetta et al., 1990; Intriligator et al., 2002), perceptual completion of shapes presented on the border between the sighted and blind field (Warrington, 1962; Torjussen, 1976), and induction of a semantic bias in interpreting auditorily presented polysemous words ("PALM") by previous visual presentation of disambiguating words ("WRIST"/“TREE") in the blind field (Marcel, 1998). Although the blind-field stimuli are task-irrelevant in this indirect type of paradigm, they can nevertheless affect the responses to, as well as the appearance and meaning of seen stimuli.

In the present study, task-irrelevant stimuli were presented to the blind field to learn whether they would affect performance in a focal attention-demanding task, and how their effect (if any) would compare to that of sighted-field distractors. Our three hemianopic participants performed a rapid serial visual presentation (RSVP) task at fixation, where 10 letters appeared in rapid succession on a local gray background. Nine letters were black. The one white letter was the target and had to be named at the end of each 10-letter trial. On $50 \%$ of trials, a task-irrelevant gray disk of negative contrast to the white background appeared. It was of high negative contrast the blind field, and of the same or a much lower negative contrast in the sighted field. These three distractor conditions were used to compare the effects of a physically prominent but subjectively invisible (blind-field) distractor to those of both a physically and 
perceptually prominent (sighted-field) distractor and a physically and perceptually innocuous (sighted-field) distractor. If visibility enhanced the distractors' disruptive effect on letter identification, the high-contrast sighted-field disk should be more effective than the low-contrast sighted-field one, and the high-contrast blind-field disk should have a weak effect at best. However, the reverse sequence should be observed if effective top-down suppression of distractors required visibility. In this latter scenario, the high-contrast disk in the blind field would be most effective, the low-contrast - sightedfield disk would follow, and the high-contrast disk in the sighted field would have no or very little effect on letter identification.

\section{MATERIALS AND METHODS PATIENTS}

Three male patients, aged 53-69 at the time of testing, participated. They had suffered vascular lesions in the territory of the right posterior cerebral artery as adults (Figure 1A), approximately 8 years before the present experiments began. The ischemic lesions of HK and WF that are responsible for their visual field defects are largely confined to V1, with HK's reaching further toward the occipital pole that is spared in WF. HK has a very small additional lesion affecting the human motion complex hMT+ on the same right side. WF's brain shows some additional damaged tissue on the lateral aspect of the right occipital lobe as well as a lesion resulting from a prior insult in the territory of the left middle cerebral area; at the time of testing the aphasic symptoms caused by this older lesion were barely noticeable. BT's single much larger lesion destroyed the right occipital lobe almost in its entirety when an arteriovenous malformation was removed; only a small remnant of ventral cerebral cortex at the pole appears spared. All three patients present with homonymous hemianopia to the left. The blindness

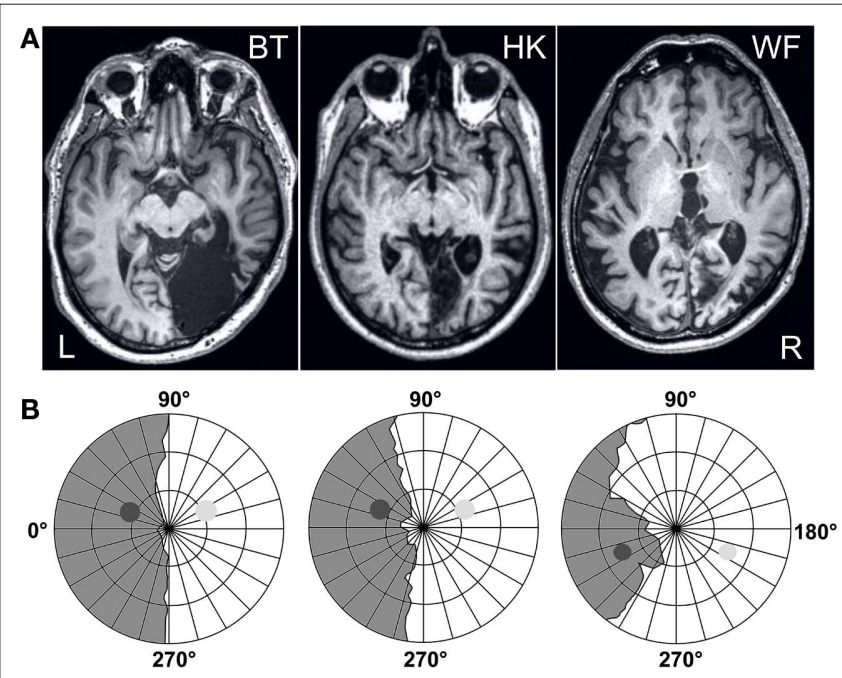

FIGURE 1 | Patients' lesions and visual fields. (A) Horizontal MRI sections reveal the lesion that damaged the right occipital lobe in all three participants. (B) The visual field of the left, contralesional eye that was used for further testing was mapped at a Tübingen perimeter with a combination of dynamic and static perimetry; gray regions indicate the blind fields. The positions of the disks used in the experiments are projected onto the plots; the high-contrast one was shown to both hemifields, the low-contrast one to the sighted field only. Concentric circles indicate $10^{\circ}, 20^{\circ}$, and $30^{\circ}$ eccentricity. is absolute in BT and WF, but $\mathrm{HK}$ recovered some very poor sight in the central portion of the upper left quadrant, and, albeit variably, had reported noting weak dark shadows in response to salient stimuli presented to this region. More peripheral regions as well as the lower quadrant remained experientially blind (see also Stoerig, 2010). All participants had extensive prior experience with tests of their residual visual functions (Stoerig et al., 2002, 2006b; Stoerig, 2010), and gave informed consent to the experiments that were approved by the University Ethics Committee.

\section{VISUAL FIELD PERIMETRY}

WF has an incomplete hemianopia, while HK and BT both have a complete hemianopia with macular sparing extending up to $5^{\circ}$ eccentricity in $\mathrm{HK}$ and $2^{\circ}$ in BT. BT originally presented with a macula-splitting hemianopia, so that his sparing represents a recovered region where sensitivity is subnormal. Plots for the left eye that was used for further testing are shown in Figure 1B. They are based on a combination of static and dynamic perimetry performed at a Tübingen perimeter (Oculus, Wetzlar, Germany). The patient's chin and forehead were supported by rests that allowed adjustment of head and eye position; a built-in telescope fitted with a reticule permitted fixation to be controlled from the examiner's side. A 116', $320 \mathrm{~cd} / \mathrm{m}^{2}$ white stimulus was moved slowly from the periphery toward the central fixation $\operatorname{dot}\left(30^{\prime}\right.$, red), or from within the blind toward the seeing part of the field; the white background had a luminance of $10 \mathrm{~cd} / \mathrm{m}^{2}$. Following delineation of visual field borders, the same stimulus was presented for $200 \mathrm{~ms}$ at closely spaced positions within the field defect, to detect any islands of vision. None of the participants responded when the stimulus was presented within the gray regions shown in Figure 1B.

\section{EXPERIMENTAL PROCEDURE RSVP task}

For testing, the participants were seated, with their head positioned by a chin and forehead rest, facing a VDU encased by a black feltlined box used to minimize stray light; the right eye was covered. Vision was normal or corrected to normal (BT and HK). At the viewing distance of $67 \mathrm{~cm}$, the monitor (Philips, refresh rate $100 \mathrm{~Hz}$ ) subtended 34.3 by $26.8^{\circ}$. At the center of its neutral white screen $\left(65 \mathrm{~cd} / \mathrm{m}^{2}\right)$, a gray square local background $\left(2^{\circ} \times 2^{\circ}\right)$ was presented. Per trial, nine black and one white capital letters (Courier New, 16 pt., $0.8^{\circ}$ high) appeared for $30 \mathrm{~ms}$ each on this local background, separated by inter-stimulus intervals of $50 \mathrm{~ms}$ (see Figure 2). The white letter appeared at serial positions 5 or 6 . At the end of each trial, the question "which letter was shown in white?" (in German)

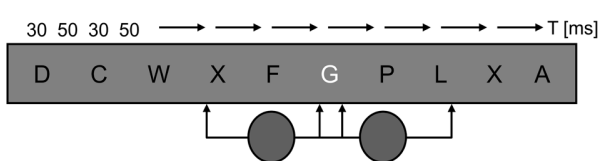

FIGURE 2 |Time course of a trial. Per trial, ten $30 \mathrm{~ms}$ letters appeared singly, separated by $50 \mathrm{~ms}$ inter-stimulus intervals, at the center of a local gray background. Only the target letter was white. On half of the trials, a task-irrelevant gray disk was presented for $190 \mathrm{~ms}$. It either preceded the target, so that disk and target offset coincided, or appeared simultaneously with the target, so that disk and target onset coincided. 
was shown on the screen. Once the participant had named the white letter, the experimenter entered the response with a keyboard, and triggered the next trial. Sixty trials were given per block. On half of these, a task-irrelevant gray disk ( $5^{\circ}$ in diameter, $190 \mathrm{~ms}$ ) was presented. It either preceded the white target letter so that its offset coincided with target offset, or appeared together with the target letter (see Figure 2); both onsets were equiprobable. In different blocks, this disk was either presented to the blind field (bf) where its position, in the upper (BT, HK) or lower (WF) quadrant, was adjusted to the individual field defect, or at the symmetric position in the sighted field (sf; see Figure 1B). A disk with a negative log contrast of $-0.6\left(16 \mathrm{~cd} / \mathrm{m}^{2}\right)$ was presented at the blind as well as the sighted-field position. In addition, a disk of much lower contrast $\left(\log -0.014 ; \sim 63 \mathrm{~cd} / \mathrm{m}^{2}\right)$ was used in the sighted field. HK and WF were also tested with a blank distractor, programmed with the same RGB values as the background, to provide a baseline for the other conditions and to ensure that drawing the disk on-screen did not interfere with the timing of the RSVP. Different distractor conditions were tested in different blocks. Per condition [blind field - high contrast (bf-hi), sighted field - high contrast (sf-hi), sighted field - low contrast (sf-lo), and blank], at least 15 blocks of trials were given in pseudo-randomized order, yielding a minimum of 900 trials per patient and condition. To avoid drawing attention to distractors, participants never received information regarding the distractor condition of the next block. E-prime 1.1 (Psychology Software Tools, Pittsburgh, PA, USA) was used as programming platform.

\section{Distractor detection}

To learn how the disk's detectability related to the effects of the distractor conditions on letter identification, a 2AFC task was conducted. Conditions were exactly as in the RSVP task, but now the letter stream only served to hold fixation. Instead of having to name the target letter, the participants were asked to press a button at the end of each trial if a disk had been presented in its course. The high-contrast disk served as target in the blind field, the low-contrast disk as target in the sighted field. Fifteen blocks of 60 trials each were given for each of these two conditions. An additional five blocks were collected with the high-contrast target in the sighted field to confirm its perfect detectability.

\section{PARTICIPANTS' EYE MOVEMENTS AND COMMENTS}

During the RSVP-task eye movements were controlled online by means of an infrared remote camera system (Iview 3.0, SensoriMotorInstruments, Teltow, Germany); as fixation had to be trained on the letter stream to solve the task, only a few randomly selected blocks were recorded. The same system was used to control fixation during the detection task (see Figure 6). At the end of the blocks, participants were asked whether they had had any impressions or wanted to comment on anything. Questions were in open format.

\section{ANALYSIS}

Based on the logfiles provided by E-prime 1.1, error rates in letter identification were first calculated per block and patient separately for each distractor condition. The resultant values were compared between bf-hi, sf-hi, sf-lo, and, in HK and WF, blank distractor conditions, using the non-parametric permutation test for dependent samples (Fisher, 1971; see Ludbrook and Dudley, 1998 for details of advantages). The false responses per patient and condition were then segregated into those given on trials without and those given on trials with distractor presentation, to learn of any trial-specific effect of distractors. Trial-specific error rates thus correspond to the proportions of all false responses falling into disk + and disk-categories and, within distractor conditions, were compared with the same permutation test. Finally, to assess effects of distractor condition on the trial-specific distractor effect, differences between error rates on trials with and trials without distractors were calculated for each block and compared between distractor conditions. Data from the two distractor-target asynchronies were collapsed because their effect on performance did not differ in any condition.

Detection performance was assessed with the $\chi^{2}$ test. $\alpha(0.05)$ was adjusted for multiple comparisons; only corrected $P$-values are reported.

\section{RESULTS}

\section{EFFECTS OF DISTRACTORS AND DISTRACTOR CONDITIONS}

With each letter presented for a mere $30 \mathrm{~ms}$, the RSVP task was demanding. Mean error rates for letter identification that include both misidentifications and "cannot tell" responses, ranged around $50 \%$ for BT and HK, whereas WF performed around $80 \%$ correct (see Figure 3); with 25 letters, chance performance was $4 \%$ correct. When the proportion of errors was calculated irrespective of whether or not a disk had been presented during a trial (i.e., across all 60 trials per block), and compared between distractor conditions, not a single difference met the statistical criterion in $\mathrm{HK}$ or WF. BT's performance however was more impaired by the highcontrast disk in the sighted field than either the low-contrast disk in the sighted field (sf-hi $>$ sf-lo: $P_{\text {corr. }}=0.035$ ) or the high-contrast disk in the blind field (sf-hi $>$ bf-hi: $P_{\text {corr. }}=0.017$ ).

Unlike the first analysis that focused on distractor conditions regardless of whether or not a disk had appeared during a trial, the second examined trial-specific effects of distractors. The

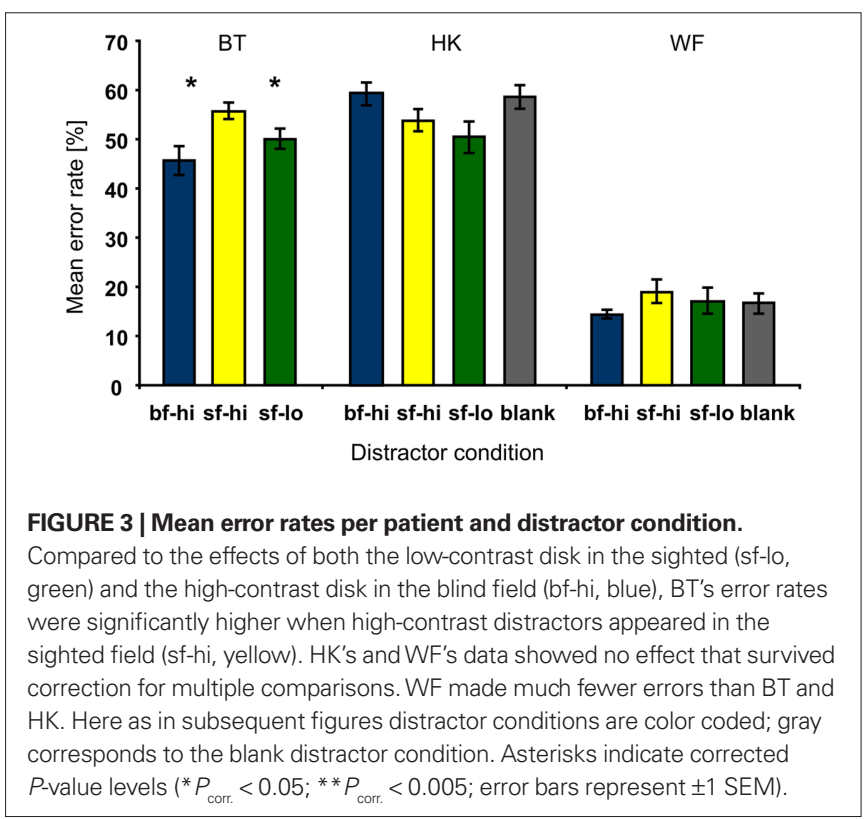


proportion of false responses per patient and condition given on trials with and on trials without distractors were calculated relative to the number of all errors; the sum of ER(disk+) and ER(disk-) thus amounts to $100 \%$. Comparing the trial-specific error rates over the blocks showed that error rates on trials with distractors were significantly higher than those on trials without distractors in the bf-hi condition only (see Figure 4). The effect was significant for $\mathrm{HK}\left(P_{\text {corr. }}=0.0007\right)$ and WF $\left(P_{\text {corr. }}=0.035\right)$ but not BT although his data showed a trend in the same direction. Trial-specific increases in error rates were also seen when the low-contrast disk was presented to the sighted hemifield of both HK and WF; however, this effect failed to survive correction for multiple comparisons even in $\mathrm{HK}$ where it was largest $\left(P_{\text {corr. }}=0.076\right)$. Like the blank disk, the $-0.6 \log$ contrast distractor presented to the sighted hemifield caused no statistically meaningful effect.

To compare the trial-specific effects between distractor conditions, the differences in error rates on trials with and without distractors $[\mathrm{ER}($ disk +$)-\mathrm{ER}($ disk-) $]$ were calculated for each block and compared for the three (BT), respectively four (HK, WF) distractor conditions. The corrected $P$-values for the comparisons are shown

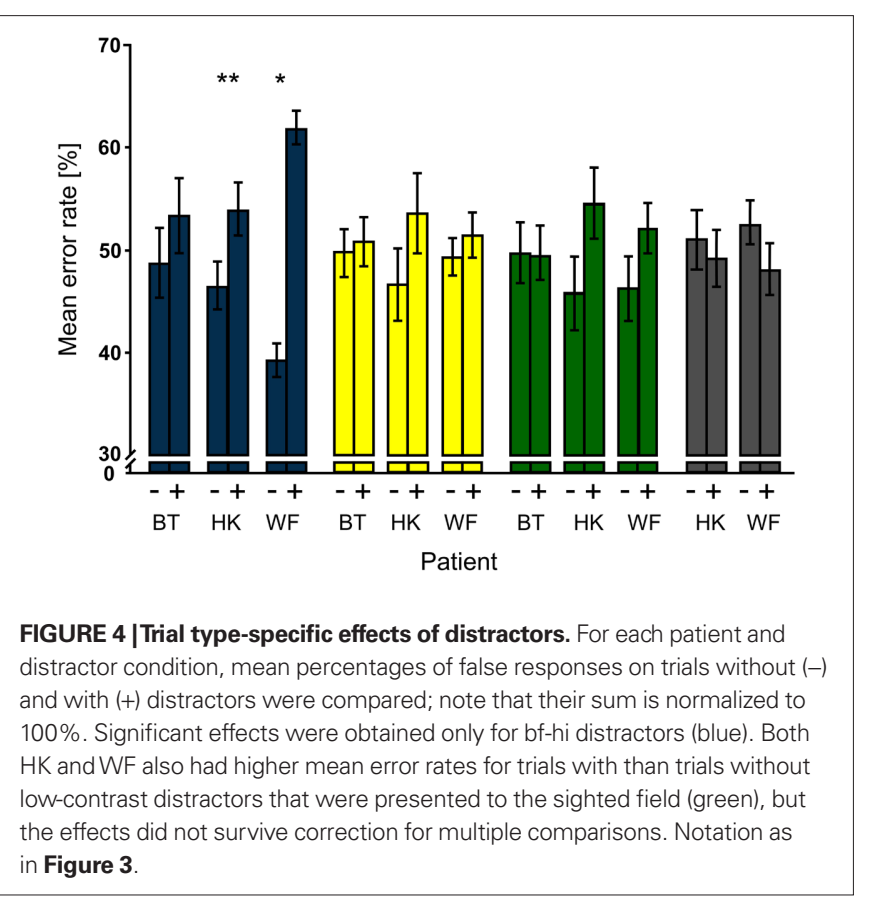

in Table 1. Note that differences were significantly larger for the bf-hi than the sf-hi or the blank distractor in HK and WF. In WF, $\mathrm{ER}($ disk+) - ER(disk-) was also significantly larger for bf-hi than sf-lo. In HK, the low-contrast distractor in the sighted field was more effective than the high-contrast sighted field and the blank distractors, so that the less visible distractors were more disruptive than the prominently visible ones whenever the conditions differed significantly. Blank and sf-hi distractors had no effect and were statistically similar. When mean differences across patients were compared between distractor conditions to learn whether any of the effects would persist across the small group, only bf-hi differed significantly from sf-hi $\left(P_{\text {corr. }}=0.024\right)$.

\section{DISTRACTOR DETECTION}

To compare the detectability of bf-hi and sf-lo disks, the participants performed a forced-choice detection task. Conditions were as in the RSVP task, but the letter stream only served to hold fixation and the disk was task-relevant. The results of these tests are straightforward. With Hit Rates exceeding False Alarm Rates by more than $20 \%$ over 900 trials, detection of the blind-field disk was highly significant in all subjects. Only HK performed worse than on other occasions (Stoerig, 2010), suggesting that the letter stream may have distracted him despite its irrelevance. As indicated by the $\chi^{2}$-values given in Figure 5 , the low-contrast disk in the sighted field was still more detectable than the high-contrast one in the blind field. Unsurprisingly, the high-contrast sighted-field disk yielded detection performance at or very close to $100 \%$ correct (not shown in Figure 5).

\section{PARTICIPANTS' COMMENTS AND EYE MOVEMENTS RSVP task}

On-line monitoring of eye movements performed by an independent observer during the RSVP task showed that saccades were confined to a small central region during the trials. Larger excursions were common between trials when the participants looked at the question on the screen or named the target letter. All participants found the task difficult. HK complained repeatedly that he often saw two white letters rather than one in single trials, and that he did not know which one to name; WF said the first two trials were always the worst; BT noted that he could often not see the left side of the letters. Error types analyzed for three randomly chosen blocks of BT's showed that this impression fits with errors committed when the target was a J, as BT said " $U$ " on four of nine presentations, and " $A$ " once; four responses were correct. The letter $\mathrm{Z}$ was named " $\mathrm{Y}$ " on seven of nine presentations, and

Table 1 | Trial type-dependent effects of distractor conditions.

\begin{tabular}{lllllll}
\hline & bf-hi $>$ sf-hi & bf-hi $>$ sf-lo & bf-hi $>$ blank & sf-lo $>$ sf-hi & sf-lo $>$ blank & sf-hi $>$ blank \\
\hline BT & ns & ns & $/$ & ns & $/$ & h \\
HK & 0.0007 & ns & 0.0170 & 0.0007 & ns & ns \\
WF & 0.0022 & 0.0002 & 0.0156 & ns & ns
\end{tabular}

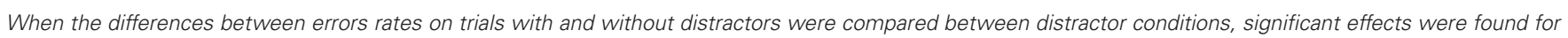

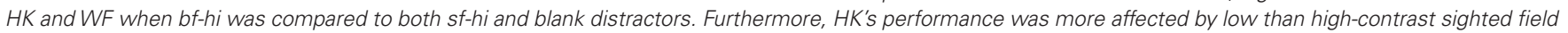

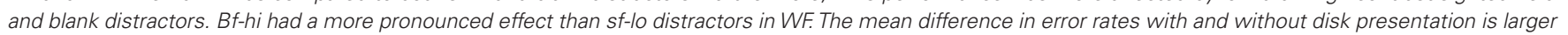
for the distractor condition named first. P-values corrected for multiple comparisons are given, I: not tested, ns: not significant at $P_{\text {corr. }}^{<} 0.05$. 


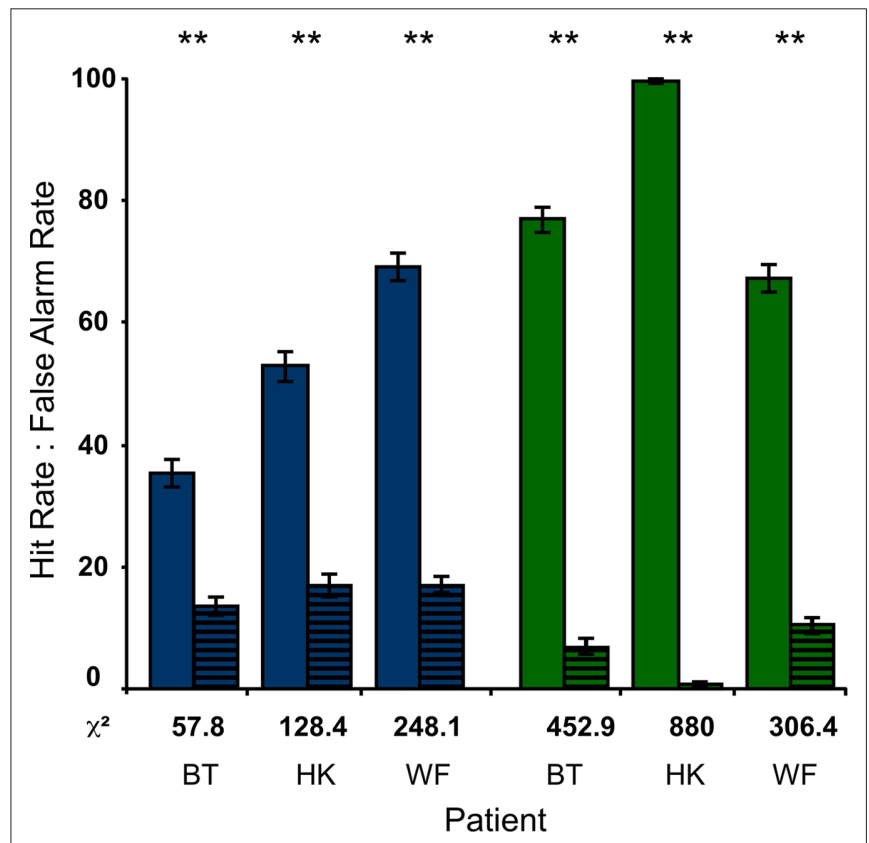

FIGURE 5 | Detection of disks. When the disks were task-relevant and the letter stream only served to hold fixation, the Hit Rates of all participants were significantly higher than the corresponding False Alarm Rates (striped). This was true for the high-contrast disk in the blind field (blue) as well as for the low-contrast disk in the sighted field (green; $P<0.001$ in all cases). As also indicated by the $\chi^{2}$-values below each pair of bars, the participants performed better in the sf-lo condition.

"N" on the remaining two. While the Z's tilted bar may account for its' being confounded with Y, errors for P: "N, C, M, W," for R: “N, M, V," and S: “D, W, U, Z, V," are not entirely explained by missing, or falsely completing the letters' left part. Nevertheless, as mentioned above, BT originally presented with a macula-splitting hemianopia; that his vision has remained less acute in the partially recovered left macular region probably explains why he did not like the letter task.

Regarding distractors, BT did not comment on them. HK, after several blocks with the high-contrast disk in the sighted field, said he had not noticed it in the preceding blocks; in contrast, the lowcontrast disk, which he at first did not seem to see either, appeared to become more prominent with continued testing. No participant reported any sensation when the blind-field disk was presented; however, on two occasions HK commented that the preceding (bf-hi) block had been "particularly difficult." Of sf-hi blocks, WF said that there was something on the right, but it did not disturb him.

\section{Detection task}

In accordance with their previous performance and high level of practise, all of the participants kept fixation very well (see Figure 6 for example). Probably because attention could now be allocated to the blind field when no disk appeared in the sighted hemifield, all patients occasionally commented on some kind of feeling or shadow that they attributed to the bf-hi disk. BT mostly said that the block had been "disastrous," that there was nothing to go by and that he pressed the "yes" button only to do me a favor. However, at the end of three consecutive blocks he

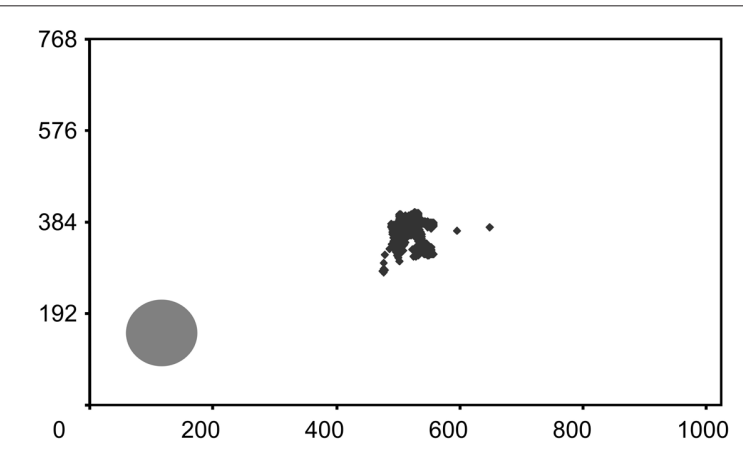

FIGURE 6 | Fixation control. WF's fixations during a standard block of 60 trials in the detection task are plotted onto an image of the monitor screen where the high-contrast disk in the blind field is also depicted. The brief fixations to the lower left occurred during the first trial and describe a curve toward the central letters. All segments corresponding to the presentations of the letter stream are given; response periods are eliminated for clarity. Numbers represent pixels. WF scored $85 \%$ correct $\left(\chi^{2}=31.1, P<0.001\right)$ and reported no sensation related to the target.

said there had been some very vague shades, and on a different occasion he described a sudden "signal" which felt like a touch which he located on his open eye. HK who in previous forcedchoice experiments often reported perceiving some weak shadow in response to blind-field targets presented in the same region, despite his excellent performance seemed to see less in the present condition, and would only occasionally say that he had "felt" very few stimuli. As he, unusually, performed worse than WF, the letter stream may have affected his performance. WF reported almost no sensations on blind-field trials, although of two blocks he said he had noticed a tiny flash to the left. All three participants were certain that they saw the low-contrast target in the sighted field, and that it was actually there when they responded by pressing the "yes" button, probably implying that this near-threshold disk was either visible or not. The high-contrast target in the sighted field was clearly visible to everyone, and missed on merely 4 of 900 trials altogether.

\section{DISCUSSION}

To learn how distractor visibility would affect performance in an attention-demanding RSVP letter identification task, task-irrelevant dark disks were presented to the sighted or blind hemifields of three hemianopes. Trial-specific distraction was non-existent for the most conspicuous sf-hi disk, but largest for the least visible bf-hi disk. The sf-lo disk whose detectability fell between that of the sf-hi and bf-hi disks, was as (HK) or less (WF) effective a distractor as the bf-hi one. Attention thus effectively suppressed the most conspicuous disk, rendering its effect statistically similar to that of a blank one, but failed when the same disk was rendered invisible in the blind field.

Global rather than trial-specific effects of distractor were found only in BT whose error rates were significantly higher in the sf-hi than either the sf-lo or the bf-hi condition. Repeated presentation of the perceptually most prominent disk apparently interfered with BT's ability to focus on the letter task. Assuming that the participants' sighted fields are normal and attention should suppress the sf-hi disk effectively as in HK and WF, we tentatively 
attribute BT's susceptibility to the sf-hi distractor condition to the letter task's annoyingly taxing his contralesionally impoverished macular vision.

\section{ATTENTION AND AWARENESS}

Attention is a selection process. Selection implies that one or more items get preferred treatment at the cost of those not so selected. If attention selected from the information of which one is aware, the deeper processing it affords would enable benefits like enhanced clarity, more detail, better reportability, more motivational impact, and better encoding for memory. Items not selected would receive much less if any such tuning. Properties of iconic memory illustrate this view, as subjects can recall the letters in any line of small arrays prompted for partial report after brief exposure. Despite reporting no more than five when a full report is requested, subjects appear to have all the letters briefly available post-offset (Sperling, 1960). If attention (instead or also) selected for awareness, it would need to enhance or deepen the processing afforded to information not yet conscious; selection would thus open the gate to sensory awareness. The phenomenon of Inattentional Blindness, in which unanticipated salient events go unnoticed when attention is engaged in a different task (Mack and Rock, 1998; Chabris and Simons, 2010), probably provides the most stunning illustration of this view. Nevertheless, a varying proportion of observers do notice the task-irrelevant items in Inattentional Blindness tasks; task difficulty and similarity between task-relevant and irrelevant objects affect this proportion (Simons and Chabris, 1999). As attention must impact information that has not reached awareness if it is to select for awareness, it might also confer deeper processing on information that does not reach awareness. Evidence supporting this possibility comes from studies of normal observers that used experimental manipulations like masking or continuous flash suppression to render stimuli invisible as well as from neurological patients with partial cortical blindness. They show that invisible stimuli may both prime sensorimotor responses to visible targets (Marzi et al., 1986; Klotz and Wolff, 1995; Intriligator et al., 2002; Schmidt, 2002; Eimer and Schlaghecken, 2003) and influence the appearance or interpretation of consciously accessible visual or auditory stimuli (Pöppel, 1986; Finlay et al., 1997; Marcel, 1998). Attentional modulation of these effects has been reported by several groups. In one of the earlier examples, Naccache et al. (2002) showed that masked numbers primed manual responses to seen target numbers only when temporal attention was allocated to the time window containing the prime-target pair. In a study by Kiefer and Brendel (2006), unrelated prime-target words elicited a "N400," an eventrelated potential signaling semantic expectancy violation, only when a cue drew attention to the masked prime words. Even adaptation to peripherally presented illusory lines was considerably improved by reducing the attentional load required by a task at fixation (Montaser-Kouhsari and Rajimehr, 2004). Work on masked response priming that related positive and negative prime-target compatibility effects to prime visibility (Eimer and Schlaghecken, 2002), was extended by Sumner et al. (2006) who cued the masked primes. They found that attention strengthened the effect of both visible and invisible primes, increased their detectability, and directly influenced the implicit priming process.
Whereas studies of normal observers have used masked primes and visible targets, visible primes were used to examine the role of spatial attention in detecting or discriminating targets presented to the affected field of a hemianope, GY, who reports awareness of sufficiently salient stimuli in his affected field (Barbur et al., 1980; Blythe et al., 1987; Zeki and ffytche, 1998; Stoerig and Barth, 2001). Kentridge et al. (1999) first showed that valid visible cues reduced response times and increased both percentage correct and aware responses to targets presented at one of two possible positions in the affected field. In a follow-up study, GY discriminated the orientation of vertical and horizontal bars presented in his affected field. Although stimulus contrast was 15\% this time, and GY reported no awareness of the targets, spatial cueing accelerated responses but did not affect accuracy (Kentridge et al., 2004). Attention may thus enhance the detectability and visibility of masked primes (Sumner et al., 2006) as well as blind-field targets (Kentridge et al., 1999) and increase the speed of discrimination responses (Kentridge et al., 2004). Regarding the present results, we do not know whether covert attention also improved performance in the disk detection task. However, attention could be oriented to the region where the disk appeared in the detection, not in the RSVP task that required it to remain trained on the central letter stream. The participants' reports suggest that visibility of the sf-lo disk was enhanced and some kind of sensation related to the bf-hi one occurred only in the detection task. In comparison to the RSVP task that focused attention elsewhere, covert attention may thus enable some lowlevel vision or "sensing" even in fields of dense cortical blindness.

\section{EFFECTS OF INVISIBLE STIMULI}

Attention can affect the processing of invisible stimuli, and invisible stimuli or stimulus features can also affect attention (see Lamme, 2003; Koch and Tsuchiya, 2007; Braun, 2009, for reviews and more examples). One example comes from a study by Rajimehr (2004, exp. 4, Figure 4) who presented single Gabor patches of imperceptibly high spatial frequency in a circular array of perceptually indistinguishable luminance defined patches. Two hundred milliseconds after the offset of the array, a visual search display appeared very briefly; subjects had to detect an upside down blue T. To learn whether the invisible Gabor singleton primed the target's position, valid (target position), invalid (contralateral to target position), and no cue conditions were compared. Whereas cues did not affect percentage correct responses, RTs were shorter on valid than invalid-cue trials. This effect, interpreted as evidence for an invisibly different singleton's attraction of covert attention, may owe more to response deceleration through the invalid cue than to response acceleration through the valid one (see Figure 4). Attraction of attention from invisible stimuli has also been demonstrated in a series of classic priming experiments. Asking whether prime-target asynchronydependent facilitatory and inhibitory priming effects (Posner and Cohen, 1984) would dissociate with practise, Lambert and Hockey (1991) presented primes and targets with equal probability to the left or right of fixation. At $50 \%$ validity primes were non-informative, and the subjects, who knew of this beforehand, had to a press key to signal the target's appearance. Over five blocks of 110 trials each, the inhibitory effect was more robust to practise and the strategic adaptations subjects may employ in its course, than the facilitatory one, provided the cue was salient. However, when the authors 
substituted a perceptually very subtle prime for the salient one, both effects persisted across blocks, indicating that perceptual salience modulates the attentional effects of peripheral cues. Whereas attentional capture from a visible stimulus can be suppressed if subjects' attention is engaged elsewhere (Yantis and Jonides, 1990), suppression fails when the stimulus is barely noticeable or invisible (Lambert and Hockey, 1991; McCormick, 1997). Tsushima et al. (2006) chose yet a different approach. To gauge how distractor visibility affects performance in an attention-demanding task, they varied the motion coherence of a random dot kinematogram (RDK) that served as task-irrelevant local background to their alphanumeric RSVP task. Results showed that their subjects' performance was disrupted most markedly when motion coherence was too low to be detected. Invisible motion coherence interfered more with the task than either zero or visible motion coherence.

By showing that a salient peripheral task-irrelevant stimulus presented to the blind field had a more disruptive trial-specific effect on letter identification than either a blank or a visible distractor in the sighted field, the present results are consonant with those reviewed above. They extend published results by demonstrating that stimuli that capture covert attention automatically need not owe their invisibility to physical weakness or experimental manipulations such as masking; a physically strong stimulus presented within a field of cortical blindness can be as captivating (Stoerig, 2006c).

\section{SPECULATION ON NEURAL MEDIATORS AND A FUNCTION OF CONSCIOUS SIGHT}

Attention can invoke baseline shifts in sensory cortex when taskrelevant stimuli are presented or even when they are expected (Chawla et al., 1999; Kastner et al., 1999). It can also bias competition between stimuli, enhancing the responses to task-relevant and suppressing those to task-irrelevant stimuli (see Desimone and Duncan, 1995; Kastner and Ungerleider, 2000; Reynolds and Chelazzi, 2004, for reviews). The first type of process may account for the enhanced visibility participants reported for the sf-lo and bf-hi disks in the detection task. Disks in the sighted field would activate early visual cortical areas contralesionally, where endogenous attention could affect the relevant neuronal populations so as to enhance the representation of the low-contrast one. The blind-field disk might activate the superior colliculus (SC) whose "detector" cells appear well-matched to its properties (Schiller and Koerner, 1971), and/or the surviving projection neurons in the dorsal lateral geniculate nucleus (dLGN); both structures have been shown to play a role in blindsight (Mohler and Wurtz, 1977; Pasik and Pasik, 1982; Schiller et al., 1990; Yoshida et al., 2008; Schmid et al., 2010). They project directly (dLGN) or indirectly (SC) to extrastriate cortical areas including V2, V4, and MT as well as the superior temporal polymodal area (STP) that continues to receive visual input via the colliculo-pulvinar route (Bruce et al., 1986; Gross, 1991). Ipsilesional area MT is a candidate cortical region, both because it remains more responsive to blind-field stimulation than other early visual cortical areas (see Bullier et al., 1994, for review) and because of the disk's sudden on- and offset. This region is anatomically intact in WF, and partially damaged by a very small lesion, but still responsive to stimulus motion in HK (Stoerig, 2006a). In BT, the lesion abuts MT, as indicated by projecting the hOC5 probability map (Malikovic et al., 2007, http://www.fz-juelich.de/inm/index.php?index = 51) onto BT's normalized structural MRI. STP is another possibility, and anatomically intact in all three participants. Effects of attention on visual areas have been found throughout the cortical hierarchy as well as in dLGN and pulvinar. As yet we do not know whether these effects persist in an occipitally lesioned system, but the still sparse behavioral data we have predict that they do.

Given that attention may have contributed to detection performance, why would it fail to engage the second type of process and suppress the high-contrast distractor in the blind field? After all, the same disk did not impair letter identification in the sighted field, and the letter identification RSVP task was the same in all distractor conditions. This high-load task should have helped attentional suppression, as a distracting moving stimulus failed to activate area MT when subjects performed an attentiondemanding task at fixation, whereas a low-load version of the same task only had a small effect on MT activation (Rees et al., 1997). Moreover, the neuronal responses the bf-hi disk evokes will most likely be weaker because the lesioned hemisphere has lost a substantial part of its retinal input; a high-contrast disk should thus induce less competition in the blind than in the sighted field if salience hinders suppression. In view of the somewhat counterintuitive effects invisible stimuli have on attention, however, the weakness of the response to the disk may indeed be the reason for its escaping suppression. Tsushima et al. (2006) not only reported that invisibly low motion coherence disrupted task performance most, they also suggested a mechanism. It invoked a lateral prefrontal area that was less activated at low coherence, suggesting that by virtue of its weakness, the low coherence RDK failed to alert the prefrontal sentry that effectively inhibited stimulus-driven activity in MT at high coherence.

Although it seems parsimonious to expect a similar mechanism to operate in our participants, we do not know whether this is the case, nor do we know whether the same brain region responds to the bf-hi disk in all patients, despite their different lesion size. BT, who has the largest lesion of our participants, would probably have the weakest neuronal responses to blind-field stimuli. Especially ventral stream areas that are already compromised by V1 lesions should be yet more affected by a lesion that includes V4, and so would areas like STP that depend on V4 in particular (Buffalo et al., 2005). If BT's comparatively poorer performance in the detection task was a consequence of yet weaker responses in ipsilesional visual cortex, his showing a tendency but no significant trial-specific effect of the bf-hi distractor might be attributed to these weakened responses. If attention failed to suppress neuronal responses to the bf-hi disk because they were too weak to alert attention in HK and WF, in BT they may simply have been too weak to not just escape suppression, but to affect letter identification in the first place. This scenario would explain the differential effect of the bf-hi disk in the participants, but functional neuroimaging of patients engaged in task-irrelevant blindsight can show whether it, or any of the conjectures above, concur with empirical data.

For the time being, the present data indicate that the blind-field distractor disrupts letter identification more effectively than the sighted-field one. While it is possible that the lesion and its sequelae damaged pathways required to mediate attentional suppression, 
the agreement between our results and those reported in normal observers make it unlikely. The latter demonstrate that task-irrelevant stimuli that are not or hardly visible can disturb responses to task-relevant ones that are visible. Under conditions of covert attention, "subjects may orient toward a signal without having first detected it" (Posner et al., 1980, p. 162), an exogenous cue can "capture attention automatically and without awareness" (McCormick, 1997, p. 168), and both invisibly low motion coherence and a disk of high negative contrast presented to a cortically blind field can disrupt performance in attention-demanding RSVP tasks, and can do so more effectively than visible motion coherence or the same disk presented to sighted fields. Following Lambert and Hockey

\section{REFERENCES}

Azzopardi, P., and Cowey, A. (2001). Motion discrimination in cortically blind patients. Brain 124, 30-46.

Barbur, J. L., Ruddock, K. H., and Waterfield, V. A. (1980). Human visual responses in the absence of the geniculocalcarine projection. Brain $103,905-928$.

Barton, J. J., and Sharpe, J. A. (1997). Motion direction discrimination in blind hemifields. Ann. Neurol. 41, 255-264.

Benson, P. J., Guo, K., and Blakemore, C. (1998). Direction discrimination of moving gratings and plaids and coherence in dot displays without primary visual cortex (V1). Eur. J. Neurosci. 10, 3767-3772.

Blythe, I. M., Kennard, C., and Ruddock, K. H. (1987). Residual vision in patients with retrogeniculate lesions of the visual pathways. Brain 110, 887-905.

Braun, J. (2009). "Attention and awareness," in The Oxford Companion to Consciousness, eds T. Bayne, A. Cleeremans, and P. Wilken (Oxford: Oxford University Press), 68-72.

Bruce, C. J., Desimone, R., and Gross, C. G. (1986). Both striate cortex and superior colliculus contribute to visual properties of neurons in superior temporal polysensory area of macaque monkey. J. Neurophysiol. 55, 1057-1075.

Buffalo, E. A., Bertini, G., Ungerleider, L. G., and Desimone, R. (2005). Impaired filtering of distracter stimuli by TE neurons following V4 and TEO lesions in macaques. Cereb. Cortex 15 , 141-151.

Bullier, J., Girard, P., and Salin, P.-A. (1994). "The role of area 17 in the transfer of information to extrastriate visual cortex," in Cerebral Cortex, Vol. 10, eds A. Peters and K. S. Rockland (New York: Plenum Press), 301-330.

Chabris, C., and Simons, D. (2010). The Invisible Gorilla and Other Ways Our Intuitions Deceive Us. New York: Random House, Crown Publishers.
Chawla, D., Rees, G., and Friston, K. J. (1999). The physiological basis of attentional modulation in extrastriate visual areas. Nat. Neurosci. 2, 671-676.

Corbetta, M., Marzi, C. A., Tassinari, G., and Aglioti, S. (1990). Effectiveness of different task paradigms in revealing blindsight. Brain 113, 603-616.

Cowey, A. (2010). The blindsight saga. Exp. Brain Res. 200, 3-24.

Cowey, A., and Stoerig, P. (1991). The neurobiology of blindsight. Trends Neurosci. 14, 140-145.

De Gelder, B., Vroomen, J., Pourtois, G., and Weiskrantz, L. (1999). Nonconscious recognition of affect in the absence of striate cortex. Neuroreport 10, 3759-3763.

Desimone, R., and Duncan, J. (1995). Neural mechanisms of selective visual attention. Annu. Rev. Neurosci. 18, 193-222.

Eimer, M., and Schlaghecken, F. (2002). Links between conscious awareness and response inhibition: evidence from masked priming. Psychon. Bull. Rev. 9, 514-520.

Eimer, M., and Schlaghecken, F. (2003). Response facilitation and inhibition in subliminal priming. Biol. Psychol. 64, 7-26.

Finlay, A. L., Jones, S. R., Morland, A. B., Ogilvie,J.A., and Ruddock,K.H.(1997). Movement in the normal visual hemifield induces a percept in the "blind" hemifield of a human hemianope. Proc R. Soc. Lond. B Biol. Sci. 264, 267-275.

Fisher, R. A. (1971). The Design of Experiments, 8th Edn. New York: Hafner Publishing.

Goebel, R., Muckli, L., Zanella, F.E., Singer, W., and Stoerig, P. (2001). Sustained extrastriate cortical activation without visual awareness revealed by fMRI studies of hemianopic patients. Vision Res. 41, 1459-1474.

Gross, C. G. (1991). Contribution of striate cortex and the superior colliculus to visual function in area MT, the superior temporal polysensory area and the inferior temporal cortex. Neuropsychologia 29, 497-515.

(1991, p. 162) who claimed that "subjects need to be clearly aware of the cue, in order for suppression of its unwanted attentional effects to occur," we suggest that attentional suppression requires (visual) awareness. If the mind is what the brain does, it does not matter whether stimulus awareness, or the processes that generate it, are necessary for attentional suppression. And if enabling attentional suppression seems a modest function for awareness, imagine life without it.

\section{ACKNOWLEDGMENTS}

It is a pleasure to thank the participants as well as Jan Breitkreuz and Eike A. Schmidt for their help in this study.

Holmes, G. (1918). Disturbances of vision caused by cerebral lesions. $\mathrm{Br}$. J. Ophthalmol. 2, 353-384.

Inouye, T. (1909). Die Sehstörungen be Schussverletzungen der kortikalen Sehsphäre. Leipzig: Engelmann.

Intriligator, J. M., Xie, R., and Barton, J. S. J. (2002). Blindsight modulation of motion perception. J. Cogn. Neurosci. 14, 1174-1183.

Kastner, S., Pinsk, M. A., De Weerd, P., Desimone, R., and Ungerleider, L. G. (1999). Increased activity in human visual cortex during directed attention in the absence of visual stimulation. Neuron 22, 751-761.

Kastner, S., and Ungerleider, L. G. (2000) Mechanisms of visual attention in the human cortex. Annu. Rev. Neurosci. 23 315-341.

Kentridge, R. W., Heywood, C. A., and Weiskrantz, L. (1999). Attention without awareness in blindsight. Proc. $R$. Soc. Lond. B Biol. Sci. 266, 1805-1811.

Kentridge, R. W., Heywood, C. A., and Weiskrantz, L. (2004). Spatial attention speeds discrimination without awareness in blindsight. Neuropsychologia 42, 831-835.

Kiefer, M., and Brendel, D. (2006) Attentional modulation of unconscious "automatic" processes: evidence from event-related potentials in a masked priming paradigm. J. Cogn. Neurosci. 18, 184-198.

Klotz, W., and Wolff, P. (1995). The effect of a masked stimulus on the response to the masking stimulus. Psychol. Res. $58,92-101$.

Koch, C., and Tsuchiya, N. (2007). Attention and consciousness: two distinct brain processes. Trends Cogn Sci. (Regul. Ed.) 11, 16-22.

Lambert, A., and Hockey, R. (1991) Peripheral visual changes and spatial attention. Acta Psychol. (Amst.) 76 149-163.

Lamme, V. A. F. (2003). Why visual attention and awareness are different. Trends Cogn. Sci. (Regul. Ed.) 7, 12-18.

Ludbrook, J., and Dudley, H. (1998). Why permutation tests are superior to $t$ and
F tests in biomedical research. Am. Stat. 52, 127-132.

Mack, A., and Rock, I. (1998). Inattentional Blindness. Cambridge, MA: MIT-Press.

Malikovic, A., Amunts, K., Schleicher, A., Mohlberg, H., Eickhoff, S. B., Wilms, M., Palomero-Gallagher, N., Armstrong, E., and Zilles, K. (2007) Cytoarchitectonic analysis of the human extrastriate cortex in the region of V5/MT+: a probabilistic, stereotaxic map of area hOc5. Cereb. Cortex 17, 562-574.

Marcel, A. J. (1998). Blindsight and shape perception: deficit of visual consciousness or of visual function? Brain 121, 1565-1588.

Marzi, C. A., Tassinari, G., Aglioti, S., and Lutzemberger, L. (1986). Spatial summation across the vertical meridian in hemianopics: a test of blindsight. Neuropsychologia 24, 749-758.

McCormick, P.A. (1997). Orienting attention without awareness. J. Exp. Psychol. Hum. Percept. Perform. 23, 168-180.

Mohler, C. W., and Wurtz, R. H. (1977) Role of striate cortex and superior colliculus of saccadic eye movements in monkeys. J. Neurophysiol. 40, 74-94.

Montaser-Kouhsari, L., and Rajimehr, R. (2004). Attentional adaptation of adaptation to illusory lines. J. Vis. 4 434-444.

Morland, A. B., Lê, S., Carroll, E., Hoffmann, M. B., and Pambakian, A. (2004). The role of spared calcarine cortex and lateral occipital cortex in the responses of human hemianopes to visual motion. J. Cogn. Neurosci. 16, 204-218.

Naccache, L., Blandin, E., and Dehaene, S. (2002). Unconscious masked priming depends on temporal attention. Psychol. Sci. 13, 416-424.

Pasik, P., and Pasik, T. (1982). Visual function in monkeys after total removal of visual cerebral cortex. Contrib. Sens. Physiol. 7, 147-200.

Payne, B. R., Lomber, S. G., Macneil, M. A. and Cornwell, P. (1996). Evidence for greater sight in blindsight following 
damage of primary visual cortex early in life. Neuropsychologia 34, 741-774.

Perenin, M. T. (1991). Discrimination of motion direction in blind fields. Neuroreport 2, 397-400.

Perenin, M. T., and Rosetti, Y. (1996). Grasping without form discrimination in a hemianopic field. Neuroreport 7, 793-797.

Pizzamiglio, L., Antonucci, G., and Francia, A. (1984). Response of the cortically blind hemifields to a moving visual scene. Cortex 20, 89-99.

Pöppel, E. (1986). Long-range colourgenerating interactions across the retina. Nature 320, 523-525.

Pöppel, E., Held, R., and Frost, D. (1973). Residual visual function after brain wounds involving the central visual pathways in man. Nature 243, 295-296.

Posner, M. I., and Cohen, Y. A. (1984). "Components of visual orienting," in Attention and Performance, Vol. $\mathrm{X}$, eds H. Bouma and H. Bouwhuis (Hillsdale, NJ: Erlbaum), 531-555.

Posner, M. I., Snyder, C. R. R., and J. Davidson, B. J. (1980). Attention and the detection of signals. J. Exp. Psychol. Gen. 109, 160-174.

Ptito, M., Johannsen, P., Faubert, J., and Gjedde,A. (1999). Activation of human extrageniculostriate pathways after damage to area V1. Neuroimage 9, 97-107.

Rajimehr, R. (2004). Unconscious orientation processing. Neuron 41, 663-673.

Rees, G., Frith, C. D., and Lavie, N. (1997). Modulating irrelevant motion perception by varying attentional load in an unrelated task. Science 287, 1616-1619.

Reynolds, J. H., and Chelazzi, L. (2004). Attentional modulation of visual processing. Annu. Rev. Neurosci. 27, 611-647.

Richards, W. (1973). Visual processing in scotomata. Exp. Brain Res. 17, 333-347.
Sanders, M. D., Warrington, E. K., Marshall, J., and Weiskrantz, L. (1974). "Blindsight": vision in a field defect. Lancet 1, 707-708.

Schiller, P. H., and Koerner, F. (1971). Discharge characteristics of single units in superior colliculus of the alert rhesus monkey. J. Neurophysiol. 34, 920-936.

Schiller, P. H., Logothetis, N. K., and Charles, E. R. (1990). Functions of the colour-opponent and broad-band channels of the visual system. Nature 343, 68-70.

Schmid, M. C., Mrowka, S. W., Turchi, J., Saunders, R. C., Wilke, M., Peters, a. J., Ye, F. Q., and Leopold, D. A. (2010). Blindsight depends on the lateral geniculate nucleus. Nature 466, 373-377.

Schmidt, T. (2002). The finger in flight: real-time motor control by visually masked color stimuli. Psychol. Sci. 13, 112-118.

Simons, D. J., and Chabris, C. (1999). Gorillas in our midst: sustained inattentional blindness for dynamic events. Perception 28, 1059-1074.

Sperling, G. (1960). The information available in brief visual presentations. Psychol. Monogr. 74, 1-29.

Stoerig, P. (2006a). "Blindsehen,” in Neuropsychologie, eds H.-O. Karnath and P. Thier (Berlin: Springer), 85-92.

Stoerig, P. (2006b). Blindsight, conscious vision, and the role of primary visual cortex. Prog. Brain Res. 155, 217-234.

Stoerig, P. (2006c). Neuroscience. The impact of invisible stimuli. Science 314, 1694-1695.

Stoerig, P. (2010). Cueless blindsight. Front. Hum. Neurosci. 3:74. doi: 10.3389/neuro.09.074.2009

Stoerig, P., and Barth, E. (2001). Low-level phenomenal vision despite unilateral destruction of primary visual cortex. Conscious. Cogn. 10, 574-587.

Stoerig, P., and Cowey, A. (1997). Blindsight in man and monkey. Brain 120, 535-559.

Stoerig, P., Zontanou, A., and Cowey, A. (2002). Aware or unaware? Assessment of cortical blindness in four men and a monkey. Cereb. Cortex 12, 565-574.

Sumner, P., Tsai, P.-C., Yu, K., and Nachev, P. (2006). Attentional modulation of sensorimotor processes in the absence of perceptual awareness. Proc. Natl. Acad. Sci. U.S.A. 103, 10520-10525.

Teuber, H.-L., Battersby, W.S., and Bender, M. B. (1960). Visual Field Defects after Penetrating Missile Wounds. Cambridge, MA: Harvard University Press.

Torjussen, T. (1976). Residual function in cortically blind hemifields. Scand. J. Psychol. 17, 320-323.

Trevethan, C. T., Sahraie, A., and Weiskrantz, L. (2007). Form discrimination in a case of blindsight. Neuropsychologia 45, 2092-2103.

Tsushima, Y., Sasaki, Y., and Watanabe, T. (2006). Greater disruption due to failure of inhibitory control on an ambiguous distractor. Science 314 1786-1788.

Warrington, E. K. (1962). The completion of visual forms across hemianopic field defects. J. Neurol. Neurosurg. Psychiatr. 25, 208-217.

Weiskrantz, L. (1986). Blindsight: A Case Study and its Implications. Oxford: University Press.

Weiskrantz, L. (1987). Residual vision in a scotoma. A follow-up study of "form" discrimination. Brain 110, 77-92.

Weiskrantz, L. (1990). Outlooks for blindsight: explicit methodologies for implicit processes. The Ferrier lecture. Proc. R. Soc. B 239, 247-278
Weiskrantz, L., Warrington, E. K., Sanders, M. D., and Marshall, J. (1974). Visual capacity in the hemianopic field following a restricted occipital ablation. Brain 97, 709-728.

Yantis, S., and Jonides, J. (1990). Abrupt visual onsets and selective attention: voluntary versus automatic allocation. J. Exp. Psychol. Hum. Percept. Perform. $16,121-134$.

Yoshida, M., Takaura, K., Kato, R., Ikeda, T., and Isa, T. (2008). Striate cortical lesions affect deliberate decision and control of saccade: implication for blindsight. J. Neurosci. 28, 10517-10530.

Zeki, S., and Ffytche, D. H. (1998). The Riddoch syndrome: insights into the neurobiology of conscious vision. Brain 121, 25-45.

Conflict of Interest Statement: The author declares that the research was conducted in the absence of any commercial or financial relationships that could be construed as a potential conflict of interest.

Received: 11 September 2010; accepted: 29 March 2011; published online: 09 April 2011.

Citation: Stoerig P (2011) Task-irrelevant blindsight and the impact of invisible stimuli. Front. Psychology 2:66. doi: 10.3389/ fpsyg.2011.00066

This article was submitted to Frontier in Consciousness Research, a specialty of Frontiers in Psychology.

Copyright $($ C 2011 Stoerig. This is an openaccess article subject to a non-exclusive license between the authors and Frontiers Media SA, which permits use, distribution and reproduction in other forums, provided the original authors and source are credited and other Frontiers conditions are complied with 